\title{
Nonlinear matched filtering for point source detection
}

\section{David Makovoz}

David Makovoz, "Nonlinear matched filtering for point source detection," Proc. SPIE 5672, Image Processing: Algorithms and Systems IV, (1 March 2005); doi: $10.1117 / 12.587283$

SPIE. Event: Electronic Imaging 2005, 2005, San Jose, California, United States 


\title{
Non-Linear Matched Filtering for Point Source Detection
}

\author{
David Makovoz \\ Spitzer Science Center, IPAC, California Institute of Technology, MS 220-6, Pasadena, CA 91125 \\ ABSTRACT
}

The task of object detection depends on the ability to suppress the noise present in images in order to increase the signal-to-noise ratio. The standard linear matched filter is the optimal filter on the assumption of the Gaussian distribution of the signal and the noise. However, as a rule the distribution of the signal in image processing is not Gaussian. The linear matched filter becomes sub-optimal. Any non-Gaussian distribution function can be closely approximated using the Gaussian Mixture Model (GMM). We use GMM to approximate the signal distribution function and derive the optimal filter by means of mean square error (MSE) minimization. The optimal non-linear filter is determined by the assumed signal distribution function. We use non-linear matched filtering for point source detection in astronomical images. We derive the GMM components by fitting the theoretical point source distribution function. The filtered images are subjected to image segmentation and subsequent point source detection. The nonlinear matched filtering has been tested with simulated data and has been shown to significantly improve the quality of point source detection. Receiver operating characteristic technique has been used to evaluate performance of various Gaussian mixtures for point source detection. This algorithm is currently used for the Spitzer Spatial Telescope.

Keywords: infrared imaging, matched filtering, non-linear filtering, non-Gaussian distributions, non-linear matched filtering, object detection, Gaussian mixture model, receiver operating characteristic

\section{INTRODUCTION}

Matched filters have been in use for a long time. Historically they have been developed as a one-dimensional detection filters for various communication systems. A matched filter can be derived ${ }^{1}$ on the basic of optimizing signal-to-noise ratio (SNR), likelihood ratio, or mean square error (MSE). Matched filters have been used extensively for object detection and tracking in two-dimensional images. Application of matched filtering in astronomy also has a long history ${ }^{2}$. At the heart of matched filtering is convolution of the input image with a reference image or a template. In astronomy such a template is the optical signature of a point source known as the point spread function. From signal detection theory we know that the linear matched filter is the optimal detector of a deterministic signal in stationary, Gaussian, additive noise. It is also the optimal filter if the signal is a distribution of sources, as long as the distribution is Gaussian.

In astronomy the sources are stars and galaxies. In this paper we deal with detection of point sources and most of the stars and galaxies are far enough to be seen as point sources by the detectors. The distribution of the point sources is very far from Gaussian. A linear filter becomes sub-optimal in this case. It is well known ${ }^{3}$ that the optimal filter in case of non-Gaussian signal or noise is non-linear. There are very few distribution functions for which it is possible to analytically derive an optimal non-linear filter. A common practice is the use of the Gaussian mixture model. A nonlinear distribution is replaced with a mixture of Gaussian components. Any non-Gaussian probability distribution function can be approximated closely by a Gaussian mixture ${ }^{4,5}$.

This paper is, to the best of our knowledge, the first published work in which a formal expression for the non-linear matched filter for two-dimensional images based on the GMM is derived. We present the results of application of nonlinear filtering to improving point source detection in astronomical images observed by the Spitzer Space Telescope ${ }^{6}$. The paper is organized as follows. In Section 2 we derive the filter. In Section 3.1 we give the basic facts on the Spitzer Space Telescope. In Section 3.2 we explained the derivation of the GMM for the point sources observed by the Spitzer Space Telescope. In Section 3.3 we describe the process of point source extraction and show the results of non-linear filtering of selected images taken by the Spitzer Space Telescope. In Section 3.4 we present a quantitative comparison of detection with linear and nonlinear filtering using the receiver operating curves (ROC) as well as the more commonly used in astronomy figures of merit of completeness and reliability. 


\section{FILTER DERIVATION}

Here we assume that image $\mathbf{s}$ observed by the detector consists of the point source contribution $\mathbf{x}$ convolved with the point response function $(\mathrm{PRF})$ and additive noise $\mathbf{n}$ :

$$
\mathbf{s}=\mathbf{H} \mathbf{x}+\mathbf{n} .
$$

PRF itself is a convolution of the optical response to an ideal point source, also known as the point spread function, with the pixel response function. $\mathbf{H}$ is a translationally invariant matrix constructed from the PRF: $\mathbf{H}_{i j}=\mathbf{H}_{j-i}=\mathrm{PRF}(i-j)$. In general, if subpixel position estimation is desired, one has to take into account the fact that the response of a pixel varies with the actual position of the point source in the pixel. Here we assume that the point sources are in the middle of the pixels. Subpixel refinement of point source positions is achieved in the process of PRF fitting of the point source, as will be explained briefly in Section 3.3.

The point source probability distribution function $f_{x}(\mathbf{x})$ is approximated with a Gaussian mixture

$$
\begin{aligned}
f_{x}(\mathbf{x}) & =P_{t o t} \sum_{\alpha=1}^{M} w_{\alpha} N_{\alpha} \exp \left(-\frac{1}{2}\left(\mathbf{x}-x_{\alpha}\right)^{T} \mathbf{C}_{\alpha}^{-1}\left(\mathbf{x}-x_{\alpha}\right)\right), \\
N_{\alpha} & =(2 \pi)^{-L / 2}\left(\operatorname{Det}\left(\mathbf{C}_{\alpha}\right)\right)^{-1 / 2} .
\end{aligned}
$$

Here $M$ is the number of terms in the mixture. Each term in the mixture is characterized by the covariance matrix $\mathbf{C}_{\alpha}$, mean $x_{\alpha}$, and weight $w_{\alpha}$. The sum of all the weights is 1 . The spatial scale $L$ is the greater of two dimensions: the average correlation length in the image and the extent of the PRF; usually $L$ is on the order of several pixels. Function $f_{x}(x)$ defines the probability density of observing a point source of flux $x$ by a detector pixel. In Section 3.2 we discuss how this function is obtained from the theoretical predictions and experimental data. $P_{\text {tot }}$ is a probability that there is any point source in the part of the sky covered by a pixel. This constant is not very well defined. It depends, among other things, on the size of the pixel, the sensitivity of the detector, the wavelength and other factors. Fortunately, it gets factored out from the final expression for the non-linear filter.

Two main contributions to the detector noise are the readout noise and the photon noise, also known as the shot noise. Even though in general it is not true, here we assume the noise to be a zero-mean Gaussian characterized by the covariance matrix $\mathbf{C}_{n}$ :

$$
\begin{gathered}
f_{n}(\mathbf{n})=N_{n} \exp \left(-\frac{1}{2} \mathbf{n}^{\mathrm{T}} \mathbf{C}_{n}^{-1} \mathbf{n}\right), \\
N_{n}=(2 \pi)^{-\mathrm{L} / 2}\left(\operatorname{Det}\left(\mathbf{C}_{\mathbf{n}}\right)\right)^{-1 / 2} .
\end{gathered}
$$

The goal of filtering is to find an estimator $\mathbf{X}(\mathbf{s})$ of the signal $\mathbf{x}$ given the observed image $\mathbf{s}$. We minimize the mean square error (MSE) of the estimator $\mathbf{X}^{\mathrm{MSE}}(\mathbf{s})$ relative to the true image $\mathbf{x}$ :

$$
\frac{\delta}{\delta \mathbf{x}} \mathrm{MSE}_{i}=\frac{\delta}{\delta \mathbf{x}} \int \mathrm{d} \mathbf{s} \int \mathrm{d} \mathbf{x} f_{n}(\mathbf{s}-\mathbf{H x}) f_{x}(\mathbf{x})\left(\mathrm{X}_{i}^{\mathrm{MSE}}(\mathbf{s})-x_{i}\right)^{2}=0
$$

where $\mathrm{MSE}_{i}$ is the mean square error for an individual pixel $i$. This leads to the following expression for the estimator $\mathbf{X}^{\mathrm{MSE}}(\mathbf{s})$ :

$$
\mathbf{X}^{\mathrm{MSE}}(\mathbf{s})=\frac{\int \mathrm{d} \mathbf{x} f_{n}(\mathbf{n}) f_{x}(\mathbf{x}) \mathbf{x}}{\int \mathrm{d} \mathbf{x} f_{n}(\mathbf{n}) f_{x}(\mathbf{x})}=\frac{\int \mathrm{d} \mathbf{x} f_{n}(\mathbf{s}-\mathbf{H} \mathbf{x}) f_{x}(\mathbf{x}) \mathbf{x}}{\int \mathrm{d} \mathbf{x} f_{n}(\mathbf{s}-\mathbf{H} \mathbf{x}) f_{x}(\mathbf{x})} .
$$


In the APPENDIX we take the integrals to arrive at the following expression

$$
\begin{aligned}
& \mathbf{X}^{\mathrm{MSE}}(\mathbf{s})=\frac{\sum_{\alpha} N_{\alpha} N_{n \alpha}^{-1} w_{\alpha}\left(\mathbf{A}_{\alpha} \mathbf{s}+\left(1-\mathbf{A}_{\alpha} \mathbf{H}\right) x_{\alpha}\right) \exp \left(-\frac{1}{2}\left(\mathbf{s}-\mathbf{H} x_{\alpha}\right)^{T} \mathbf{C}_{n}^{-1}\left(1-\mathbf{H} \mathbf{A}_{\alpha}\right)\left(\mathbf{s}-\mathbf{H} x_{\alpha}\right)\right)}{\sum_{\alpha} N_{\alpha} N_{n \alpha}^{-1} w_{\alpha} \exp \left(-\frac{1}{2}\left(\mathbf{s}-\mathbf{H} x_{\alpha}\right)^{T} \mathbf{C}_{n}^{-1}\left(1-\mathbf{H} \mathbf{A}_{\alpha}\right)\left(\mathbf{s}-\mathbf{H} x_{\alpha}\right)\right)} ; \\
& \mathbf{A}_{\alpha}=\mathbf{C}_{\alpha} \mathbf{H}^{T}\left(\mathbf{C}_{n}+\mathbf{H} \mathbf{C}_{\alpha} \mathbf{H}^{T}\right)^{-1} ; \\
& N_{n \alpha}=(2 \pi)^{-L / 2}\left(\operatorname{Det}\left(\mathbf{C}_{\alpha}+\mathbf{H}^{\mathrm{T}} \mathbf{C}_{n} \mathbf{H}\right)\right)^{-1 / 2} .
\end{aligned}
$$

Here superscript $T$ denotes matrix transposition. In the case of a single zero-mean component in the GMM with the covariance matrix $\mathbf{C}_{1}$ the above expression is reduced to the conventional expression of the linear matched filter

$$
\mathbf{X}^{\mathrm{MSE}}(\mathbf{s})=\mathbf{C}_{1} \mathbf{H}^{T}\left(\mathbf{C}_{n}+\mathbf{H} \mathbf{C}_{1} \mathbf{H}^{T}\right)^{-1} \mathbf{s}
$$

For the practical application we simplified the above expression assuming spatially uncorrelated noise and GMM components:

$$
\begin{aligned}
& C_{\alpha}(i, j)=\delta_{i j} \sigma_{\alpha}^{2} ; \\
& C_{n}(i, j)=\delta_{i j} \sigma_{n}^{2} .
\end{aligned}
$$

With this simplification expression (4) takes on the following final form:

$$
\mathbf{X}^{\mathrm{MSE}}(\mathbf{s})=\frac{\sum_{\alpha} w_{\alpha}\left(\frac{\sigma_{\alpha}}{\sqrt{\sigma_{n}^{2}+\sigma_{\alpha}^{2} H^{2}}} \mathbf{H s}+\frac{\sigma_{n}}{\sqrt{\left(\sigma_{n}^{2}+\sigma_{\alpha}^{2} H^{2}\right)}} x_{\alpha}\right) \exp \left(-\frac{\left(\mathbf{s}-\mathbf{H} x_{\alpha}\right)^{2}}{2\left(\sigma_{n}^{2}+\sigma_{\alpha}^{2} H^{2}\right)}\right)}{\sum_{\alpha} \frac{\sqrt{\sigma_{n}^{2}+\sigma_{\alpha}^{2} H^{2}}}{\sigma_{\alpha}} w_{\alpha} \exp \left(-\frac{\left(\mathbf{s}-\mathbf{H} x_{\alpha}\right)^{2}}{2\left(\sigma_{n}^{2}+\sigma_{\alpha}^{2} H^{2}\right)}\right)}
$$

The corresponding simplification of the linear matched filter (6) takes on the following form:

$$
\mathbf{X}^{\mathrm{MSE}}(\mathbf{s})=\frac{\sigma_{1}^{2}}{\sigma_{n}^{2}+\sigma_{1}^{2} H^{2}} \mathbf{H} \mathbf{s} .
$$

In the next section we describe application of the non-linear filter to some real and simulated astronomical images. We show the significant improvement achieved by using the non-linear matched filter compared the linear matched filter.

\section{APPLICATION OF NONLINEAR FILTERING}

\subsection{Basic Facts on the Spitzer Space Telescope}

We applied the non-linear filter derived in the previous section to astronomical images taken by the recently launched Spitzer Space Telescope ${ }^{6}$. Spitzer Space Telescope is a space-borne, cryogenically-cooled infrared observatory capable of studying objects ranging from our Solar System to the distant reaches of the Universe. Spitzer is the final element in NASA's Great Observatories Program, and an important scientific and technical cornerstone of the Astronomical Search for Origins Program. The Observatory carries an 85-centimeter cryogenic telescope and three cryogenically cooled science instruments capable of performing imaging and spectroscopy in the 3.6 to 160 micron range. Spitzer was launched on a Delta $7920 \mathrm{H}$ from Cape Canaveral into an Earth-trailing heliocentric orbit in August 2003. Spitzer carries three instruments on board for taking images at different wavelengths. Table 1 provides basic information on the instruments, detector type and size, and the wavelength. 
The individual detector frames are combined into one mosaic image ${ }^{7}$. Mosaic images cover areas much larger than the individual frames. The biggest mosaic images created up-to-date cover $\sim 10$ sqr deg. Normally, observations are taken with some redundancy, where the same area of the sky is covered by anywhere from 2 to several hundred images. This means that the mosaic images also have a higher signal to noise ratio than the individual frames. Consequently, detection of point sources is performed in the mosaic images.

\begin{tabular}{|l|l|l|l|l|}
\hline Instrument Name & Detector Type & Wavelength(micron) & Detector Size (pixel) & Pixel Size(arcsec) \\
\hline IRAC & In:Sb & 3.6 & $256 \times 256$ & $1.2 \times 12$ \\
\hline IRAC & In:Sb & 4.5 & $256 \times 256$ & $1.2 \times 12$ \\
\hline IRAC & Si:As & 5.8 & $256 \times 256$ & $1.2 \times 12$ \\
\hline IRAC & Si:As & 8.0 & $256 \times 256$ & $1.2 \times 12$ \\
\hline IRS & Si:As & 15 & $33 \times 45$ & $1.8 \times 1.6$ \\
\hline MIPS & Si:As & 24 & $128 \times 128$ & $2.4 \times 2.4$ \\
\hline MIPS & Ge:Ga & 70 & $32 \times 32$ & $4.9 \times 4.9$ \\
\hline MIPS & Ge:Ga & 160 & $20 \times 2$ & $15 \times 15$ \\
\hline
\end{tabular}

Table 1 Spitzer imaging measurement functionality. IRAC stands for InfraRed Array Camera, IRS stands for InfraRed Spectrograph, MIPS stands for Multiband Imaging Photometer for Spitzer.

\subsection{GMM Fitting of the Point Source Flux Density Distribution Function}

In order to apply the non-linear filter (8) we need to use a realistic point source flux distribution function and fit it with a Gaussian mixture. Usually, astronomers deal with differential point source counts, where the fluxes of point sources are binned and histogrammed. These point source counts have to be normalized to produce point source flux distribution functions. The difficulty with using point source counts is that they depend on many factors. Theory predicts different point source counts for galactic sources ${ }^{8}$ (which are stars) and extragalactic sources ${ }^{9}$ (which are galaxies themselves). Point source counts depend on the wavelength ${ }^{10,11}$ for which the sources are observed. In the future we can work on filter optimization by deriving different distribution functions for different wavelengths and investigate how sensitive the final results of point source detection are to the exact details of the point source distribution function. For the purpose of this paper we assume that point source detection is not very sensitive to the exact shape of the distribution function. We combine the point source distribution functions ${ }^{8,9}$ to obtain a generalized distribution function that has the main features of those distribution functions. It is shown in Figure 1 as a dashed line.

The point source distribution function is fitted with 4 Gaussian components. We use simulated annealing technique to perform fitting. The parameters of the Gaussian mixture components are given in Table 2. Figure 1 shows the GMM fit to the generalized distribution function as the solid line.

\begin{tabular}{|l|l|l|l|}
\hline \multicolumn{1}{|c|}{$\alpha$} & $\sigma_{\alpha}$ & $x_{\alpha}$ & $w_{\alpha}$ \\
\hline 1 & 0.01 & 0.31 & 0.14 \\
\hline 2 & 0.11 & 0.41 & 0.16 \\
\hline 3 & 0.89 & 0.62 & 0.19 \\
\hline 4 & 9.8 & 0.73 & 0.51 \\
\hline
\end{tabular}


Table 2 Parameters of the GMM fit of the point source distribution function.

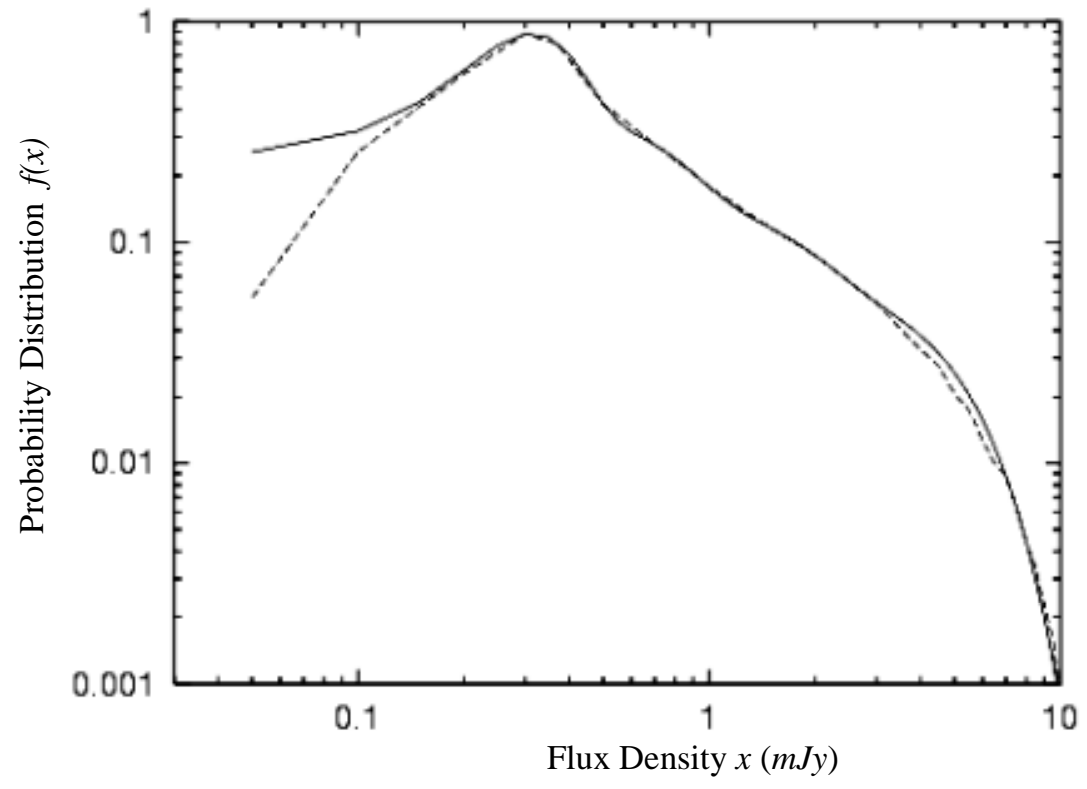

Figure 1 Shown here with the dashed line is the generalized point source flux density distribution function. The GMM fit is shown with the solid line. 4 Gaussian components are used.

A Jansky $(J y)$ is a unit of flux density defined as $10^{-26} \mathrm{~W} /\left(\mathrm{m}^{2} \mathrm{~Hz}\right)$.

Once we have the parameters of the GMM, we can compute the response function of the nonlinear filter (8). Since the input as well as the output of the filter are vectors, the response function cannot be easily visualized. We reduce them to scalars by considering the case of the PRF reduced to the delta function:

$$
X^{\mathrm{MSE}}(s)=\frac{\sum_{\alpha} w_{\alpha}\left(\frac{\sigma_{\alpha}}{\sqrt{\sigma_{n}^{2}+\sigma_{\alpha}^{2}}} s+\frac{\sigma_{n}}{\sqrt{\left(\sigma_{n}^{2}+\sigma_{\alpha}^{2}\right)}} x_{\alpha}\right) \exp \left(-\frac{1}{2} \frac{\left(s-x_{\alpha}\right)^{2}}{\sigma_{n}^{2}+\sigma_{\alpha}^{2}}\right)}{\sum_{\alpha} \frac{\sqrt{\sigma_{n}^{2}+\sigma_{\alpha}^{2}}}{\sigma_{\alpha}} w_{\alpha} \exp \left(-\frac{1}{2} \frac{\left(s-x_{\alpha}\right)^{2}}{\sigma_{n}^{2}+\sigma_{\alpha}^{2}}\right)}
$$

We plot (9) in Figure 2 for the GMM defined in Table 1as a function of $\mathrm{SNR}_{\mathrm{GMM}}$ :

$$
\mathrm{SNR}_{\mathrm{GMM}}=10 \lg \sum_{\alpha} \frac{w_{\alpha} \sigma_{\alpha}^{2}}{\sigma_{n}^{2}}
$$

There is a non-zero output even for $s=0$. It comes from the imperfection of the model that says that there is a non-zero probability to have a point source at every pixel even for zero input. Then there is the suppression region. The range of the suppression depends on the noise power. It represents the fact that the observed signal $s$ there is mostly noise. In the limit of $s \rightarrow \infty$ the filter becomes linear with the slope given by the well known Wiener filter for the highest component of the GMM. 


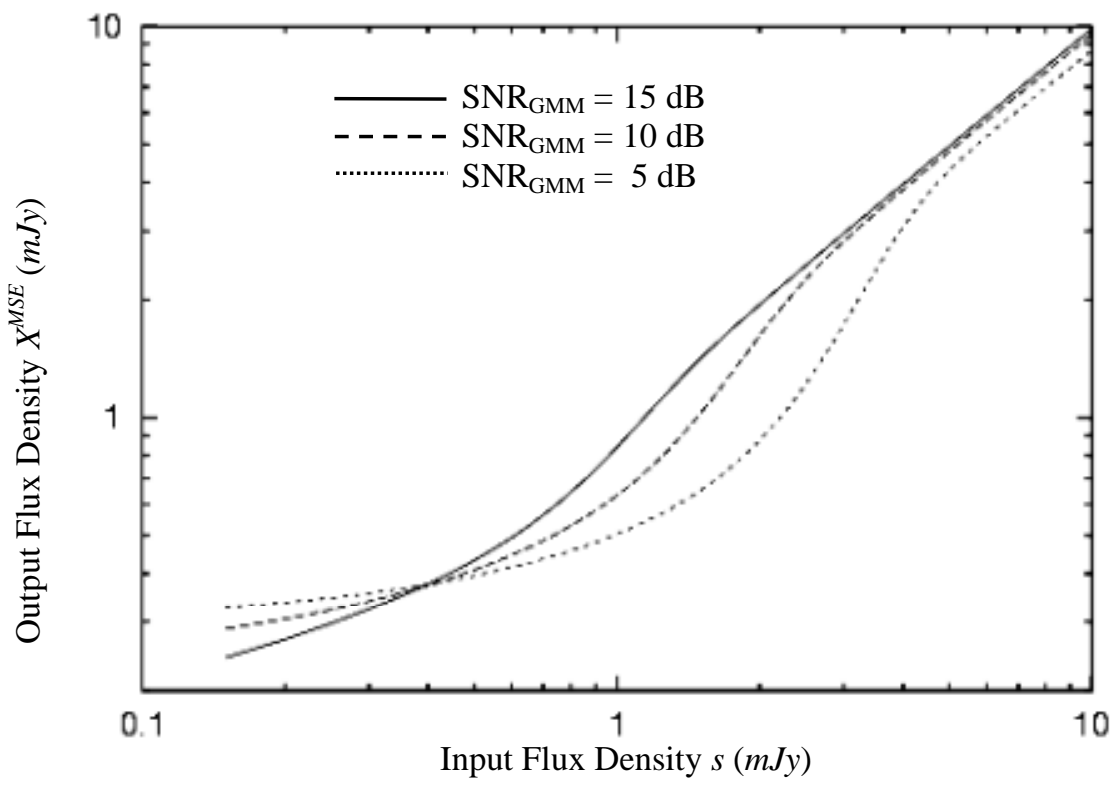

Figure 2 The response function of the non-linear filter (8) for 3 values of $\mathrm{SNR}_{\mathrm{GMM}}$.

\subsection{Point Source Extraction in Spitzer Images}

In this section we briefly describe the process of point source extraction employed by the Spitzer software package MOPEX $^{7}$. It consists of four stages: image filtering, image segmentation, point source detection, point source PRF fitting. The final product is the list of point sources with the refined positions and fluxes. In this paper we report the results of point source extraction for IRAC 5.8 micron and MIPS 70 micron images.

The ingredients of the non-linear filter (8) in addition to the GMM components (Table 2) are the PRF images. The PRF images for IRAC 5.8 micron and MIPS 70 micron are presented in Figure 3. IRAC 5.8 micron PRF has the FWHM (full-width half-maximum) $=1.5$ pixels $=1.8$ arcsec; MIPS 70 micron PRF has the FWHM $=5$ pixels $=25$ arcsec. These PRF's are also used for PRF fitting.
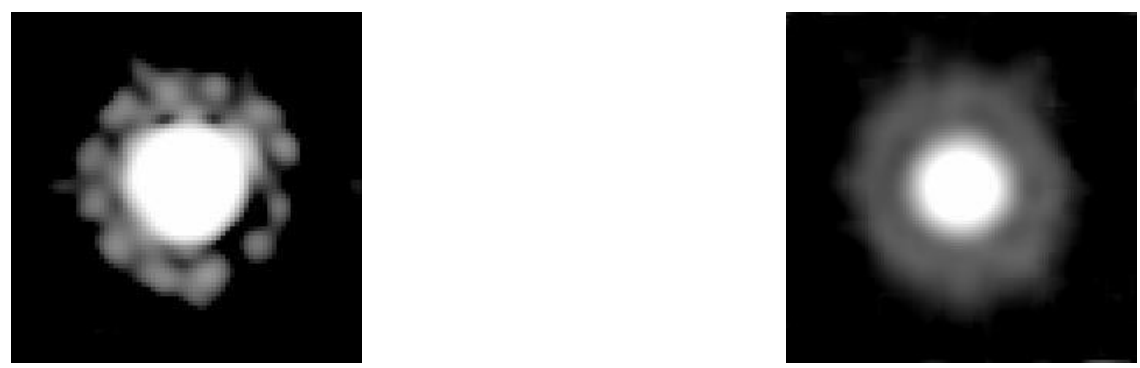

Figure 3 Here we show the PRF images for IRAC 5.8 micron (left) and MIPS 70 micron (right).

One more component of the non-linear filter is pixel noise $\sigma_{n}$. This quantity is estimated from the data. A noise image is created. A sliding window is moved across the input image and for each pixel the width of the distribution of the pixel values in the window centered on that pixel is computed. This is done on the assumption of Gaussian noise.

Non-linear filtering suppresses the noise and brings up the source in the filtered images. In Figure 4 we show a portion of an input mosaic image and the corresponding filtered image for IRAC 5.8 micron. This image is characterized by a low background level and high density of point sources. In Figure 5 we show a portion of an input mosaic image and the 
corresponding filtered image of MIPS 70 micron. This image is characterized by a much higher background and lower density of point sources. Convolution with the PRF causes a noticeable smearing of the point sources in the MIPS 70 micron image, since the PRF there has a significant FWHM. The smearing, however, is not of concern, since the filtered images are used for detection only.
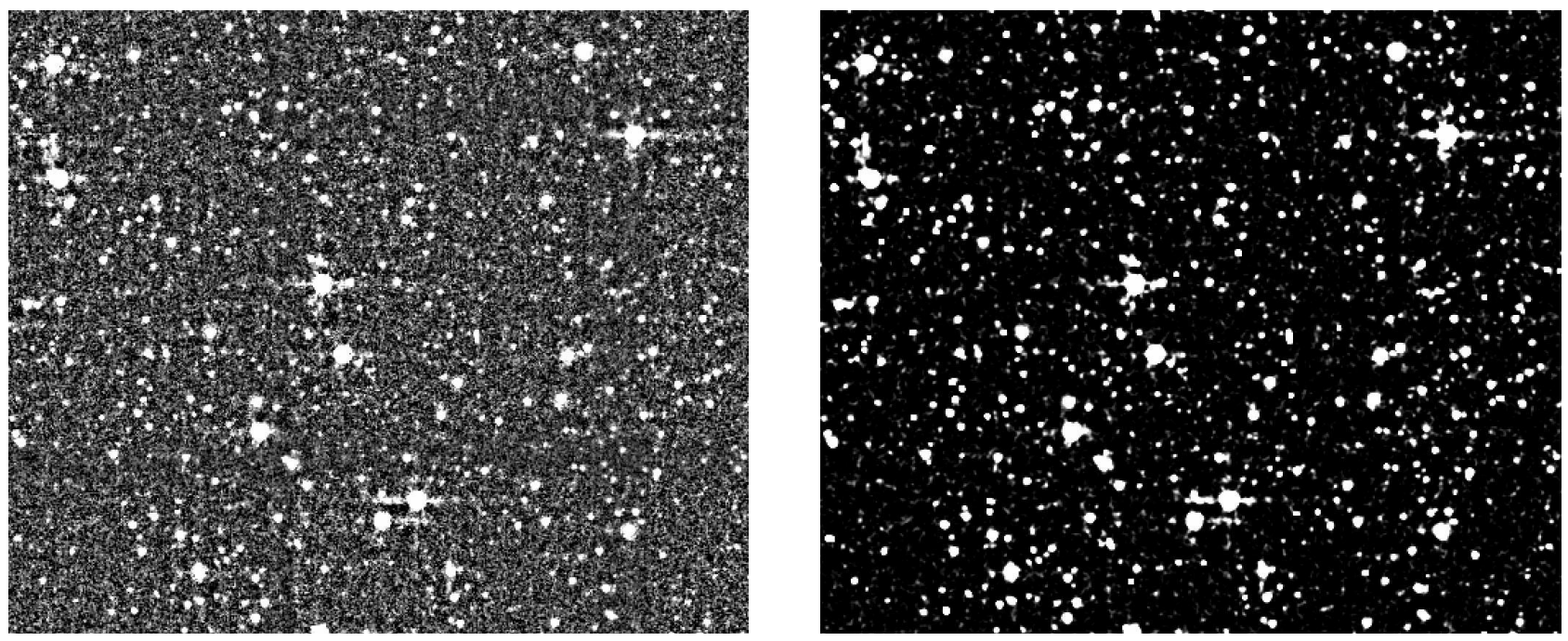

Figure 4. Shown here on the left is a portion of a mosaic image for IRAC 5.8 micron. On the right is the mosaic image filtered with non-linear filtering. The size of the image is $\sim 500$ pixels $\mathrm{x} 400$ pixels or $0.17^{\circ} \mathrm{x} 0.13^{\circ}$.
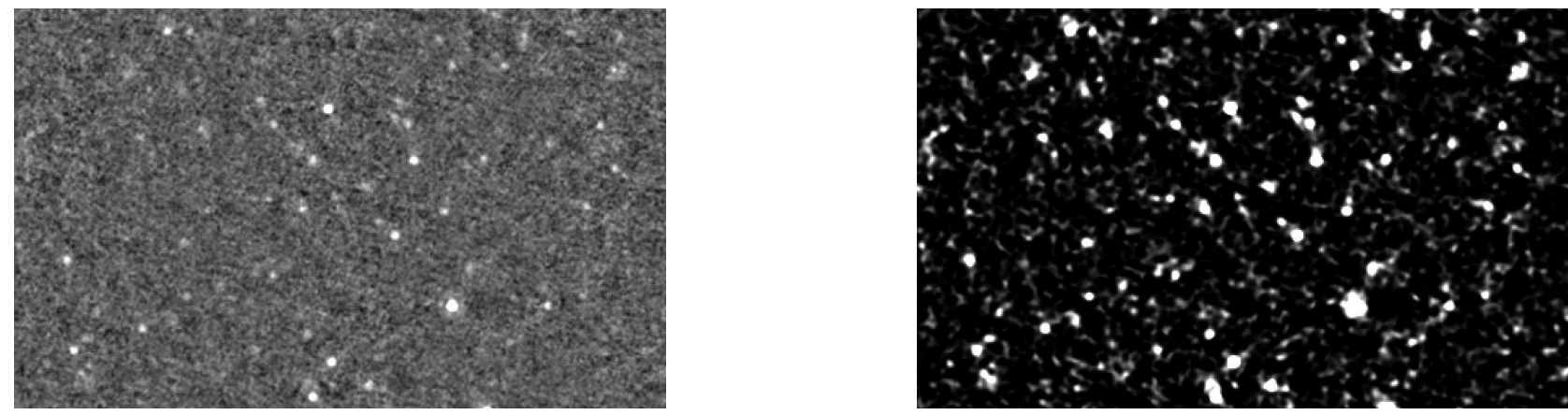

Figure 5 Shown here on the left is a portion of a mosaic image for MIPS 70 micron. On the right is the mosaic image filtered with non-linear filtering. The size of the image is $\sim 450$ pixels $\mathrm{x} 300$ pixels or $0.5^{\circ} \times 0.33^{\circ}$.

Once the image is filtered image segmentation is performed. In the filtered images contigous clusters of pixels above a user specified threshold are found. For each cluster the centroid is computed. The centroid is the preliminary position of the point source.

At this point a list of detected sources is produced. In order to accurately estimate the flux of point sources PRF fitting is performed. The result of PRF fitting is a list of point sources with improved positions and fluxes. By binning the fluxes differential point source counts are obtained. The distribution of the extracted point source fluxes is compared with the point source flux distribution function used to generate the Gaussian mixture. Ideally, one can now rederive the GMM by fitting the observed point source distribution function and then do filtering again until the process converges. In practice, however, we have not done this, partially because of the involved complexity and partially because, as was mentioned before, the results of detection is not very sensitive to the details of the non-Gaussian distribution function used to derive the non-linear filter. 

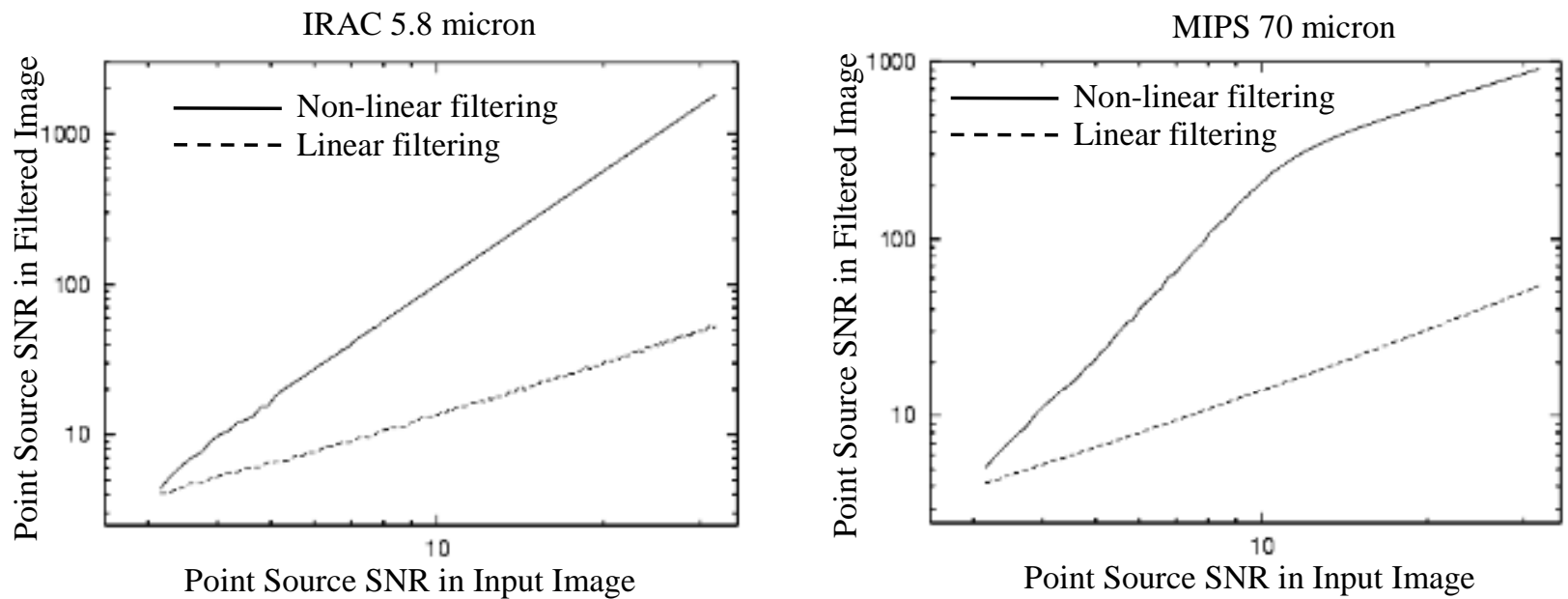

Figure 6 Shown here are plots of SNR of point sources in the filtered images versus SNR of the same sources in the input image.

Even though in deriving the expression for the non-linear filter the SNR was not explicitly optimized, the SNR is greatly enhanced as a result of filtering. We define SNR of the point sources here as the ratio of their flux measured within a suitably defined aperture to the noise level measured in the vicinity of the point source. The noise image described above is used for this purpose. We want to emphasize that SNR here, according to the accepted convention in astronomy, is not defined on the log scale, as opposed to the one defined in (10). A comparison of the SNR of the point sources in the input image and the SNR of the same sources in the filtered image is a measure of the effectiveness of filtering. We plot in Figure 6 the SNR of the point sources in the non-linear and linear filtered images versus the SNR of the same source in the original mosaic image. The SNR of the point sources in the non-linear filtered images is significantly higher than the SNR in the linear filtered images.

\subsection{Validation of Non-linear Filtering with Simulated Data}

So far we have discussed point source detection and extraction in real images. In order to perform quantitative comparison of the detection using different filtering methods one has to compare the results of detection with the "truth". This can be done when extraction is performed on simulated images. For this purpose a set of images was simulated for each instrument of the Spitzer Space Telescope. The point source flux density distribution was simulated using the model ${ }^{9}$. Truth sources were assigned both random and correlated positions. Truth sources were convolved with the PRF. The images of the point sources were added to the background, which was simulated using the model ${ }^{12}$. Subsequently, point source extraction was performed as described in the previous section. The extracted point sources were matched with the true point sources based on their positions and fluxes. Point source extraction was performed in images simulated for IRAC 5.8 micron and MIPS 70 micron.

Usually in target detection the receiver operating curves (ROC) are used to determine the optimal threshold and to compare various filtering methods. The threshold referred to here is the one used for detecting clusters, as described in the previous section. PRF fitting is not very sensitive to the way the filtering is performed, because the fitting is done in the original image. In Figure 7 we plot the false alarm rate versus the hit rate as a parametric function of the detection threshold. The false alarm rate is defined as a percentage of falsely detected point sources. The hit rate is defined as a percentage of correct detections. Here we only included the point sources with SNR > 3 . 
IRAC 5.8 micron

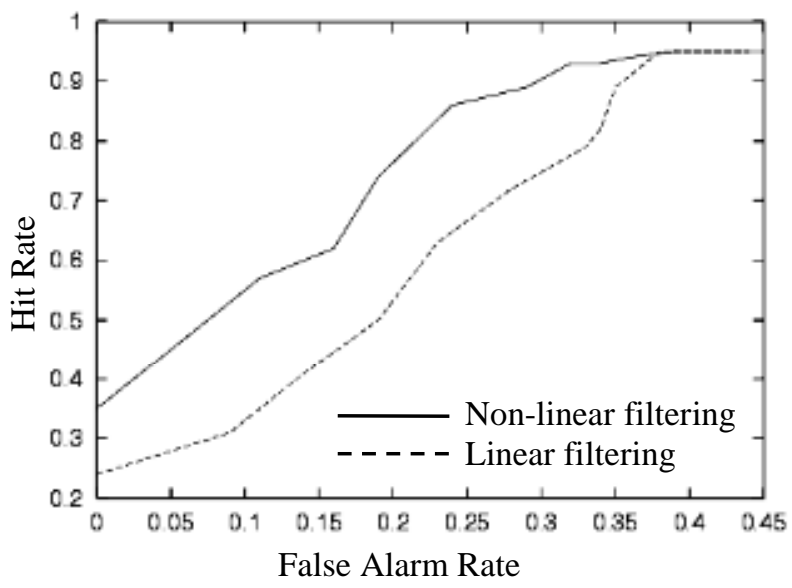

MIPS 70 micron

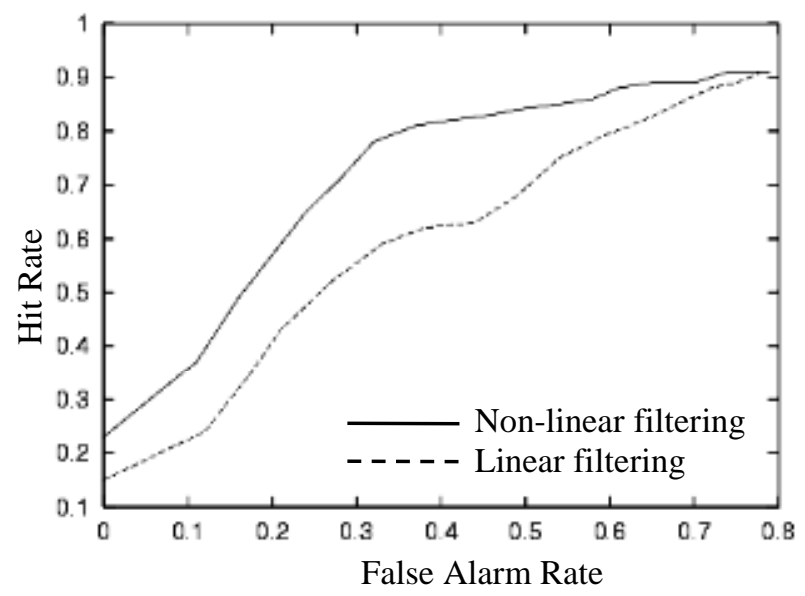

Figure 7 Hit rate versus false alarm rate for point sources with SNR $>3$.

For point source extraction in astronomy such cumulative figures of merit as the hit rate and false alarm rate for all sources is too general and does not provide enough detailed information. The conventional figures of merit in astronomy are the differential completeness $(C)$ and reliability $(R)$ computed as functions of the point source fluxes. The thresholding in this case is done at a level low enough to detect the faintest interesting sources. All the point sources are binned according to their fluxes. For each flux bin $x$ completeness $C(x)$ is defined as the ratio of the number of the matched sources $N_{\text {match }}(x)$ to the number of true sources $\mathrm{N}_{\text {true }}(\mathrm{x})$. Reliability $R(x)$ is defined as the ratio of the number of the matched sources $N_{\text {match }}(x)$ to the number of detected sources $N_{\text {det }}(x)$.

$$
C(x)=\frac{N_{\text {match }}(x)}{N_{\text {true }}(x)} ; \quad R(x)=\frac{N_{\text {match }}(x)}{N_{\text {det }}(x)} .
$$

In Figure 8 we plot the differential completeness and reliability computed from the results of the point source extraction in the simulated images. The non-linear filtering produces superior results compared to linear filtering.

\section{CONCLUSION}

In this paper we derived an expression for a non-linear matched filter. The non-linear filter is derived for non-Gaussian signal using the Gaussian mixture model. We derived the parameters of a 4-component Gaussian mixture by fitting the generalized point source flux density distribution function. We presented application of the non-linear matched filter to point source extraction in astronomical images observed by the Spitzer Space Telescope. The SNR of the point sources in the images produced by the non-linear matched filtering is much higher than that of the point sources in the images produced by the linear matched filtering. We presented a comparison of completeness and reliability of point source detection in simulated images for the non-linear matched filtering compared to the linear matched filtering. The nonlinear filtering has been shown to produce superior results.

I would like to thank the MIPS and IRAC Instrument Support Teams in general, and David Frayer in particular, for reducing the data and making the MIPS 70 micron PRF. I also would like to thank David Shupe for creating the simulated images used in this work. This work was carried out at the Spitzer Science Center, with funding from NASA under contract 1407 to the California Institute of Technology and the Jet Propulsion Laboratory. 
IRAC 5.8 micron
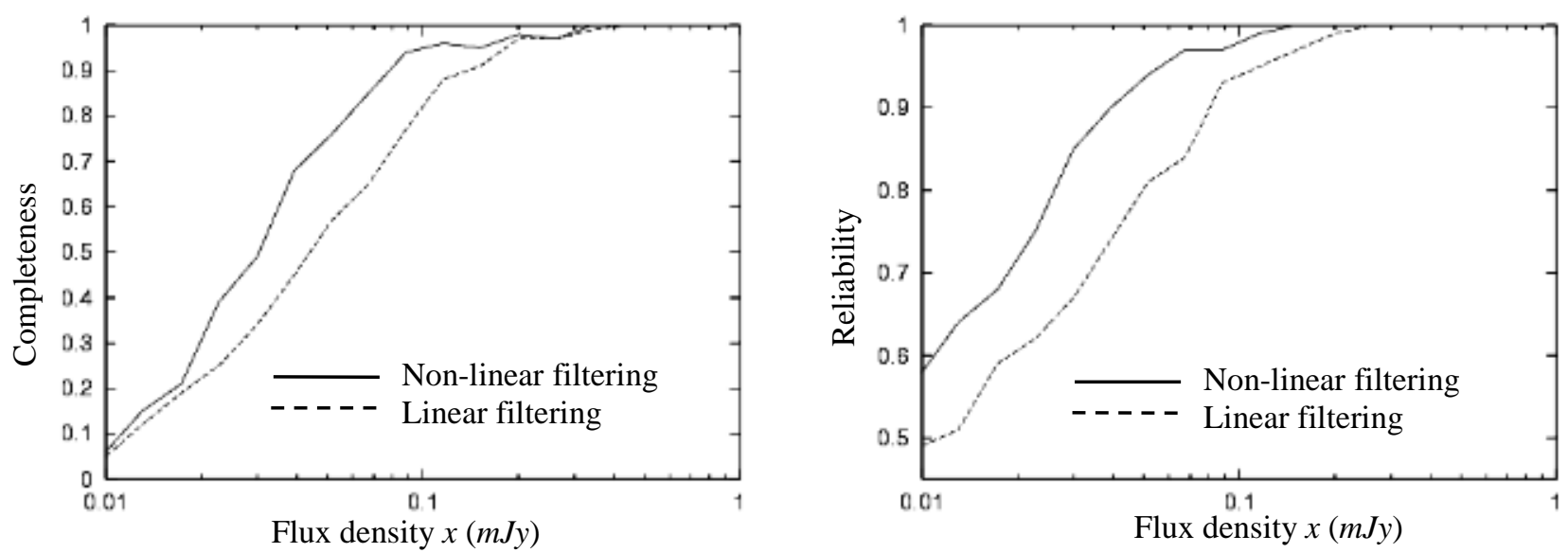

MIPS 70 micron
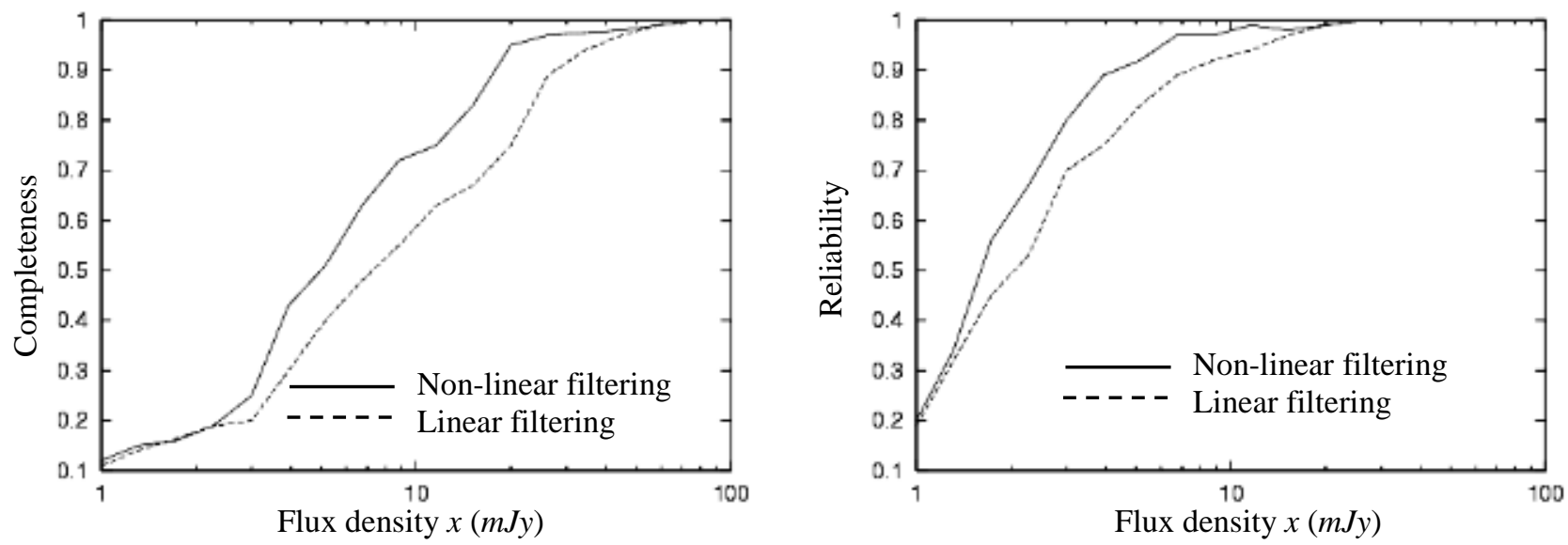

Figure 8 Shown are plots of differential completeness and reliability as functions of point source flux density.

\section{REFERENCES}

1. C.E. Cook and M. Bernfeld, Radar Signals, Academic Press, New York, 1965

2. H.C. Andrews, Computer Techniques in Image Processing, Academic Press, New York, 1970

3. E.R. Dougherty, Random Processes for Image and Signal Processing, SPIE/IEEE Series on Imaging Science \& Engineering, IEEE Press, 1998.

4. D.M. Titterington, A.F.M. Smith, and U.E. Makov, Statistical Analysis of Finite Mixture Distribution, Wiley, New York, 1985.

5. G.J. McLachlan and K.E. Basford, Mixure Models: Inference and Application to Clustering, Marcel Dekker, New York, 1988.

6. http://www.spitzer.caltech.edu/

7. D. Makovoz, I. Khan, "Mosaicking with MOPEX", Astronomical Data Analysis Software and Systems VI, 132, in ASP Conf. Ser., San Francisco.

8. M. Cohen, “A Model of the 2-35 micron Point Source Infrared Sky “, Astrophysical Journal, 105, p.1860, 1993. 
9. C. Hu et al., "Emission features and source counts of galaxies in the mid-infrared", Astrophysical Journal, 508, p.576, 1998.

10. F.R. Marleau et al., "Extragalactic Source Counts at 24 Microns in the Spitzer First Look Survey", Astrophysical JournalLetters, 154, p.66, 2004.

11. H. Dole et al., "Far-infrared Source Count at 70 and 160 Microns in Spitzer Deep Surveys", Astrophysical JournalLetters, 154, p.87, 2004.

12. Kelsall et al., "The COBE Diffuse Infrared Background Experiment Search for the Cosmic Infrared Background: II. Model of the Interplanetary Dust Cloud", Astrophysical Journal, 508, p.44, 1998

\section{APPENDIX}

Here we take the integrals in equation (4). Both integrals have the common part:

$$
f_{n}(\mathbf{s}-\mathbf{H x}) f_{x}(\mathbf{x})=\sum_{\alpha} w_{\alpha} N_{\alpha} N_{n} \exp \left(-\frac{F_{\alpha}}{2}\right) .
$$

After substituting expression (2) for $f_{x}$ and expression (3) for $f_{n}$ we get the following for $F_{\alpha}$

$$
\begin{aligned}
F_{\alpha} & =\mathbf{y}_{\alpha}^{T} \mathbf{C}_{\alpha}^{-1} \mathbf{y}_{\alpha}+\left(\mathbf{s}-\mathbf{H} \mathbf{y}_{\alpha}-\mathbf{H} x_{\alpha}\right)^{T} \mathbf{C}_{n}^{-1}\left(\mathbf{s}-\mathbf{H} \mathbf{y}_{\alpha}-\mathbf{H} \mathbf{x}_{\alpha}\right) \\
\mathbf{y}_{\alpha} & \equiv \mathbf{x}-x_{\alpha}
\end{aligned}
$$

This expression can be transformed to separate a quadratic form in $\mathbf{y}$ and the $\mathbf{y}$-independent part

$$
\begin{aligned}
& F_{\alpha}=\mathbf{z}_{\alpha}^{T}\left(\mathbf{C}_{\alpha}^{-1}+\mathbf{H}^{T} \mathbf{C}_{n}^{-1} \mathbf{H}\right) \mathbf{z}_{\alpha}+\left(\mathbf{s}-\mathbf{H} x_{\alpha}\right)^{T} \mathbf{B}_{\alpha}\left(\mathbf{s}-\mathbf{H} x_{\alpha}\right) ; \\
& \mathbf{z}_{\alpha}=\mathbf{y}_{\alpha}-\mathbf{A}_{\alpha}\left(\mathbf{s}-\mathbf{H} x_{\alpha}\right) ; \\
& \mathbf{A}_{\alpha}=\mathbf{C}_{\alpha} \mathbf{H}^{T}\left(\mathbf{C}_{n}+\mathbf{H} \mathbf{C}_{\alpha} \mathbf{H}^{T}\right)^{-1} ; \\
& \mathbf{B}_{\alpha}=\mathbf{C}_{n}^{-1}\left(1-\mathbf{H} \mathbf{A}_{\alpha}\right) .
\end{aligned}
$$

After this decomposition the integrals are taken easily. The denominator is equal to

$$
\int \mathrm{d} \mathbf{x} f_{n}(\mathbf{s}-\mathbf{H} \mathbf{x}) f_{x}(\mathbf{x})=N_{n} \sum_{\alpha} N_{\alpha} w_{\alpha} \exp \left(-\frac{1}{2}\left(\mathbf{s}-\mathbf{H} x_{\alpha}\right)^{T} \mathbf{B}_{\alpha}\left(\mathbf{s}-\mathbf{H} x_{\alpha}\right)\right) \Psi_{\alpha},
$$

where the integral $\Psi_{\alpha}$ is equal to

$$
\Psi_{\alpha}=\int \mathrm{d} \mathbf{z}_{\varepsilon} \exp \left(-\frac{1}{2} \mathbf{z}_{\alpha}^{T}\left(\mathbf{C}_{\alpha}^{-1}+\mathbf{H}^{\mathrm{T}} \mathbf{C}_{\mathrm{n}}^{-1} \mathbf{H}\right)^{-1} \mathbf{z}_{\alpha}\right)=(2 \pi)^{-L / 2}\left(\operatorname{Det}\left(\mathbf{C}_{\alpha}+\mathbf{H}^{\mathrm{T}} \mathbf{C}_{n} \mathbf{H}\right)\right)^{-1 / 2} \equiv N_{n \alpha}^{-1}
$$

Therefore the denominator is equal to

$$
\int \mathrm{d} \mathbf{x} f_{n}(\mathbf{s}-\mathbf{H} \mathbf{x}) f_{x}(\mathbf{x})=\sum_{\alpha} N_{n} N_{\alpha} N_{n \alpha}^{-1} \exp \left(-\frac{1}{2}\left(\mathbf{s}-\mathbf{H} x_{\alpha}\right)^{T} \mathbf{B}{ }_{\alpha}\left(\mathbf{s}-\mathbf{H} x_{\alpha}\right)\right) .
$$

The numerator is equal to 


$$
\begin{aligned}
& \int \mathrm{d} \mathbf{x} f_{n}(\mathbf{s}-\mathbf{H} \mathbf{x}) f_{x}(\mathbf{x}) \mathbf{x}= \\
& N_{n} \sum_{\alpha} N_{\alpha} w_{\alpha}\left(\mathbf{z}_{\alpha}+x_{\alpha}+\mathbf{A}_{\alpha}\left(\mathbf{s}-\mathbf{H} x_{\alpha}\right)\right) \exp -\frac{1}{2}\left(\left(\mathbf{s}-\mathbf{H} x_{\alpha}\right)^{T} \mathbf{B}_{\alpha}\left(\mathbf{s}-\mathbf{H} x_{\alpha}\right)\right) \Psi_{\alpha}= \\
& N_{n} \sum_{\alpha} N_{\alpha} w_{\alpha}\left(x_{\alpha}+\mathbf{A}_{\alpha}\left(s-\mathbf{H} x_{\alpha}\right)\right) \exp -\frac{1}{2}\left(\left(\mathbf{s}-\mathbf{H} x_{\alpha}\right)^{T} \mathbf{B}_{\alpha}\left(\mathbf{s}-\mathbf{H} x_{\alpha}\right)\right) \Psi_{\alpha}= \\
& \sum_{\alpha} N_{n} N_{\alpha} N_{n \alpha}^{-1}\left(x_{\alpha}+\mathbf{A}_{\alpha}\left(\mathbf{s}-\mathbf{H} x_{\alpha}\right)\right) \exp \left(-\frac{1}{2}\left(\mathbf{s}-\mathbf{H} x_{\alpha}\right)^{T} \mathbf{B}_{\alpha}\left(\mathbf{s}-\mathbf{H} x_{\alpha}\right)\right)
\end{aligned}
$$

which leads to equation (5). 